\title{
Further comments on the evidence for post-retinal storage
}

\author{
THEODORE E. PARKS 1 \\ UNIVERSITY OF CALIFORNIA, DAVIS
}

A critical re-examination of the perceptual reports given in the experiments described by Haber and Nathanson reveals confirmation of the essential elements of the phenomena previously reported by Parks. Specifically, it is argued that a moving stimulus viewed through a narrow stationary opening gives rise to percepts containing more stimulus information than is, in fact, available to the eye at any instant.

Parks (1965) described the percepts that occurred when a simple stimulus pattern was moved behind a narrow viewing-slit in a fixed, otherwise-opaque, screen. If the movement was rapid, there was tendency for the figure to be seen as a whole in the vicinity of the slit even though only a part of the figure was actually visible at any moment. Furthermore, there was a strong tendency for such a figure to be seen as foreshortened in the direction of its movement. These phenomena would seem to have important implications concerning the post-retinal storage and integrative abilities of the visual system.

More recently, Haber and Nathanson (1968) described an extensive series of studies centering around the same basic experimental conditions. Contrary to the earlier results, their Ss never reported seeing a stimulus as a whole. Whole percepts were reported only in a contrast condition in which the stimulus figure was stationary with respect to the eye and the screen containing the viewing-slit moved rapidly over the figure.

Their paper would seem, however, to be seriously marred by a failure to interpret completely one other highly important aspect of their Ss' reports and, as a result, by a misevaluation of the theoretical implications of their observations. That is, Haber and Nathanson failed to recognize the importance of the combination of their Ss' reports of shrinkage in the figure together with their reports that "all they could see of the figure [at any instant] was what was visible in the slit" (Haber \& Nathanson, pp. 352 and 354). While the latter reports fall short of reporting single percepts of the whole figure, the essential point is that such reports, in combination with reports of foreshortening, clearly imply that Ss had, at any instant, a percept of more than what was actually available at any instant within theviewing-slit. For example, using a pattern of concentric circles as a stimulus, if the center ring is reported as an ellipse which is only as wide as the slit, then clearly the $S$ is reporting that he seems to see at least all of that part of stimulus in one, instantaneous percept. While this is only a hypothetical example, the same logic must apply to any part of any stimulus pattern that is reported as appearing narrower than the width of the slit when, objectively, that part of the pattern is broader than the slit. Thus, the observations of Haber and Nathanson are at variance with those of the original investigation seemingly only in degree. That is, only with respect to how much of a given figure is represented in a single percept. It is, of course, quite reasonable to expect that this latter dependent variable will be a function of the specific properties of the stimulus (form, contrast, and illumination) all of which still await careful exploration.

Finally, if post-retinal storage is involved here (a point that will be firmly established only when careful observations of eye movements are made coincidentally with instances of the phenomena), then the relative ease with which whole percepts are obtained when the slit is moved over a stationary figure is readily understandable. In the latter condition, presumably both retinal and post-retinal after-effects would contribute to the percept.

In brief, Haber and Nathanson's Ss reported more than they saw and, thus, were saying a good deal more than the authors realized.

\section{REFERENCES}

HABER, N. H., \& NATHANSON, L. S. Post-retinal storage? Some further observations on Parks' camel as seen through the eye of a needle. Perception and Psychophysics, 1968, 3, 349-355.

PARKS, T. E. Post-retinal visual storage. American Joumal of Psychology, $1965,78,145-147$.

\section{NOTE}

1. Address: Department of Psychology, University of California, Davis, California 95616.

(Accepted for early publication August 8, 1968.) 